\title{
Research on Noise Reduction of 3.6 MW Evaporative Cooling Wind Motor Induced by Electromagnetic and Two-Phase Flow Resonance Based on Stator Optimization
}

\author{
Ziran Cheng ${ }^{1}\left(\mathbb{D}\right.$, Lin Ruan ${ }^{2, *}\left(\mathbb{D}\right.$, Shoudao Huang ${ }^{1, *}$ and Jie Yang ${ }^{2}$ \\ 1 College of Electrical and Information Engineering, Hunan University, Changsha 410082, China; \\ chengziran@mail.iee.ac.cn \\ 2 Institute of Electrical Engineering (IEE) of Chinese Academy of Sciences (CAS), \\ University of Chinese Academy of Sciences, Beijing 100190, China; yangiie@mail.iee.ac.cn \\ * Correspondence: rosaline@mail.iee.ac.cn (L.R.); hsd1962@hnu.edu.cn (S.H.)
}

check for updates

Citation: Cheng, Z.; Ruan, L.; Huang,

S.; Yang, J. Research on Noise

Reduction of 3.6 MW Evaporative

Cooling Wind Motor Induced by

Electromagnetic and Two-Phase Flow

Resonance Based on Stator

Optimization. Processes 2021, 9, 669.

https://doi.org/10.3390/pr9040669

Academic Editors: Myung-Seop Lim and Matti Lehtonen

Received: 8 March 2021

Accepted: 7 April 2021

Published: 10 April 2021

Publisher's Note: MDPI stays neutral with regard to jurisdictional claims in published maps and institutional affiliations.

Copyright: (c) 2021 by the authors. Licensee MDPI, Basel, Switzerland. This article is an open access article distributed under the terms and conditions of the Creative Commons Attribution (CC BY) license (https:// creativecommons.org/licenses/by/ $4.0 /)$.

\begin{abstract}
In this paper, the vibration frequency of the stator of 3.6 MW fully immersed evaporative cooling permanent magnet semi-direct drive generators (ECPMSDDGs) was analyzed based on the fluid-structure coupling theory and solved by finite element analysis (FEA) simulation. The resonance noise reduction research under the typical working condition induced by two-phase flow and electromagnetic force was studied based on the method of structural optimization. In this paper, a structural optimal design method for the stator of the 3.6 MW ECPMSDDGs was presented. First of all, the frequency and characteristics of electromagnetic force of 3.6 MW ECPMSDDGs under the rated power were analyzed. Secondly, the frequency and characteristics of two-phase flow boiling vibration were analyzed based on the bubble oscillation theory of the two-phase flow and the experiment. Thirdly, the wet modal natural frequency of the stator core cooling structure was analyzed based on the fluid-structure coupling theory and FEA. Finally, the natural wet mode vibration frequency of the stator cooling structure of the 3.6 MW ECPMSDDGs was improved based on the structure optimization. This optimization method could reduce the resonance noise of evaporative cooling motor induced by electromagnetic and two-phase flow. The optimization results showed that the natural wet mode frequency of the stator could be improved by optimizing the radial flow groove and supporting beam under the condition that the effective length of the stator core remained unchanged during the optimization. The noise simulation result showed that the resonance noise of 3.6 MW ECPMSDDGs induced by electromagnetic and two-phase flow could be reduced after the structural optimization.
\end{abstract}

Keywords: evaporative cooling; permanent magnet semi-direct drive generators; wet mode analysis; stator structure optimization; resonance noise reduction

\section{Introduction}

Recently, wind energy has been the fastest growing and most commercially promising renewable energy. It has broad development prospects due to its low cost and vast resources [1]. One or two step-up speed gearboxes can usually be adopted by permanent magnet semi-direct drive generators (PMSDDGs) to reduce the volume of the motor. It is a compromised choice of the direct drive wind power generators and the multi-stage growth wind power generators. The permanent magnet synchronous motor (PMSM) is widely used in the wind power system due to its characteristics such as high power density, high efficiency, simple structure, reliable operation conditions without excitation windings and DC excitation power supply. The cooling conditions will worsen more and more with the increasing capacity of wind generators and the evaporative cooling technology could significantly improve the heat dissipation efficiency of the motor [2], which is very suitable 
for the application in large capacity wind generators and has a good prospect in the large capacity offshore wind generators in the future.

Vibration and noise are the common phenomena in the operation of wind generators, which will be detrimental to the operation of generators and the capture of wind energy. The electromagnetism and two-phase flow excitation sources will cause vibration and noise when the motor is operating in PMSDDGs. Recently, many scholars have carried out corresponding researches about these problems to reduce the motor vibration and noise. The vibration and noise of the PMSMs can be normally reduced by stator skewing, rotor skewing, electromagnetic parameter optimizing and the stator structural optimizing [3]. For the studies of vibration and excitation force of two-phase flow, Seryakov studied the boiling resonance phenomenon in heat pipes and analyzed the pulsation characteristics of the heat pipe under boiling state [4]. Unno and Yuki et al. studied the relationship between plate vibration and boiling heat transfer coefficient under the condition of subcooled boiling heat transfer based on the natural vibration frequency of the heating plate [5]. Elkholy and Kempers carried out experiments on the geyser boiling phenomenon in two-phase flow circulation, and studied the influence of parameters such as fluid state and heat flux on fluid vibration [6]. However, few researches have analyzed and tested the boiling condition aimed at special cooling structure of large capacity evaporative cooling motor. The vibration and noise reduction methods of permanent magnet wind motor mainly include the electromagnetic force and structure optimizing [7-9] at present. Islam and Jahirul studied the vibration of wind turbine caused by cogging torque, torque ripple and radial gear force [10]. Shinagam and Ramakrishna developed a novel methodology to reduce the vibrations by inserting a steel coil spring in turnbuckle D shackle assembly of $200 \mathrm{~kW}$ wind turbine generators [11]. Zhu studied the torsional vibration reduction in drive-train to reduce the stress and avoid potential resonances with wind turbines system [12]. However, few researches have studied the noise and vibration reduction method based on the stator cooling structure optimization.

According to the literature researched, it can be known that there are few researches on the reduction of vibration and noise of large capacity evaporative cooling wind motor. The common motor noise reduction studies only consider the electromagnetic force excitation. In fact, evaporative cooling permanent magnet semi-direct drive generators (ECPMSDDGs) are the novel type of the wind motor, which has a different operating environment from the ordinary wind motor and has both of these problems at the same time during the process of operation. Evaporative cooling motor has the unique cooling flow structure which could be optimized in the process of noise reduction. In this paper, the frequency of electromagnetic and two-phase flow excitation force of 3.6 MW ECPMSDDGs has been studied. A new stator cooling structure of 3.6 MW ECPMSDDGS was designed based on structure optimization, which could reduce the resonance noise of the stator caused by both electromagnetic and two-phase flow vibration excitation. The optimization process of stator cooling structure can not only provide references for the resonance noise reduction design of the fully immersed ECPMSDDGs cooling structure, but can also provide a basis for the frequency optimization design for other types of motor stator cooling structures of different working conditions.

\section{Analysis of Electromagnetic Excitation Force of Stator}

\subsection{Stator Model of Fully Immersed Evaporative Cooling Motor}

Different from the traditional wind generators with outer rotor structure and air cooling system, the 3.6 MW ECPMSDDGs adopted inner rotor structure and fully immersed evaporative cooling natural circulation cooling system in stator. The motor structure is shown in Figure 1.

As shown in Figure 2, the cooling chamber of the stator was composed of isolation sleeve, sealing end cover and casing, etc. The stator core and windings were placed in a closed cavity filled with liquid cooling medium. Segment structure was adopted by the stator core and radial flow grooves of the stator core were filled with liquid cooling 
medium. The liquid cooling medium flowed through the end of the winding and the radial flow grooves. The cooling medium was transported to the condenser by density difference for condensing after absorbing the heat in the stator core and vaporizing. The temperature rise of the stator core and the inner winding could be effectively reduced by the evaporative cooling process.

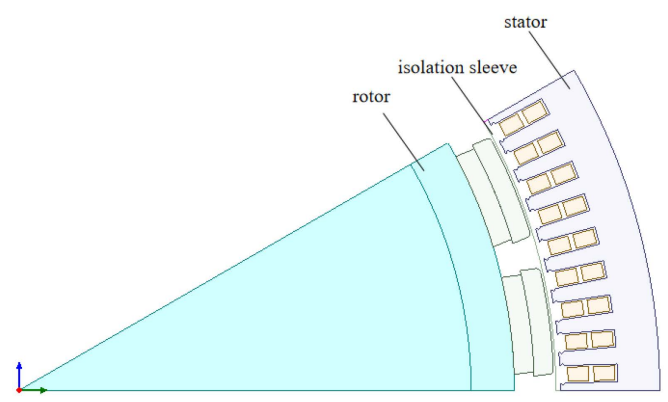

Figure 1. Structure of 3.6 MW fully immersed evaporative cooling permanent magnet semi-direct drive generators (ECPMSDDGs) motor.

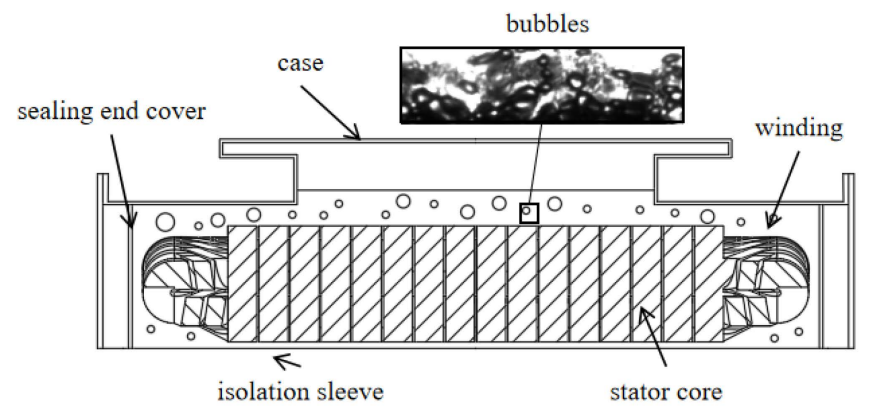

Figure 2. Stator cooling structure of 3.6 MW fully immersed ECPMSDDGs.

\subsection{Analysis of Electromagnetic Excitation Force of 3.6 MW ECPMSDDGs}

The electromagnetic noise of the PMSM is caused by alternating electromagnetic field and is mainly related to the characteristics of electromagnetic field, forced vibration parts and the shape and size of the motor space. The main electromagnetic parameters of 3.6 MW fully immersed ECPMSDDGs are shown in Table 1.

Table 1. Main electromagnetic parameters of 3.6 MW fully immersed ECPMSDDGs.

\begin{tabular}{cc}
\hline Parameters & Value \\
\hline Rated power $/(\mathrm{kW})$ & 3600 \\
Phase number & 3 \\
Rated phase voltage $/(\mathrm{V})$ & 381 \\
Length of stator core $/(\mathrm{mm})$ & 1020 \\
Effective length of stator core $/(\mathrm{mm})$ & 855 \\
Rated speed $/(\mathrm{rpm})$ & 350 \\
Rated frequency/(Hz) & 70 \\
Stator slot number & 108 \\
Number of poles & 24 \\
Embrace of permanent magnet & 0.75 \\
Length of air gap/(mm) & 7.5 \\
Stator inner diameter $/(\mathrm{mm})$ & 1250 \\
Total loss $/(\mathrm{kW})$ & 124 \\
\hline
\end{tabular}

The fundamental magnetic field and a series of harmonic magnetic fields will be contained in the air gap magnetic fields during the operation of the motor. The electromagnetic force will be generated by the interaction of these magnetic fields [13]. This 
electromagnetic force could be decomposed into radial and tangential force. Compared with the tangential force, the contribution of radial electromagnetic force to the noise is higher [14]. With the increase of harmonic frequency of the electromagnetic force, the amplitude of electromagnetic force will decrease. The number of pole-pairs in the rotor magnetic harmonic magnetic field is:

$$
\mu=(2 r+1) P, r=0,1,2,3 \ldots
$$

where $\mu$ represents the number of pole-pairs in the rotor magnetic harmonic magnetic field. $P$ represents the number of pole-pairs of the motor.

The number of pole-pairs in the stator winding harmonic magnetic field is:

$$
v=\left(\frac{6 k}{d}+1\right) P, k=0, \pm 1, \pm 2 \ldots
$$

where $d$ is the denominator of the improper fraction of the fractional-slot.

The order of electromagnetic radial force waves generated by the interaction between the stator harmonics and the rotor harmonics is $n=u \pm v$. The excitation frequency of the radial force wave of order $n$ is:

$$
f_{n}=(\mu \pm 1) f_{0}
$$

where $f_{0}$ is the rated frequency.

The order of radial force waves is shown in Table 2. It can be seen from the table that the main frequency of the radial force waves of the synchronous motor are even times of the rated frequency.

Table 2. Space order of radial electromagnetic waves.

\begin{tabular}{cccccc}
\hline$\mu$ & 12 & -24 & 48 & -60 & 84 \\
$v$ & $24 / 2$ & $-12 / 2$ & $60 / 2$ & $-48 / 2$ & $96 / 2$ \\
\hline \multirow{2}{*}{12} & $0 / 0$ & $36 / 0$ & $-36 / 0$ & $72 / 0$ & $-72 / 0$ \\
& $48 / 4$ & $12 / 4$ & $84 / 4$ & $-24 / 4$ & $120 / 4$ \\
\multirow{2}{*}{36} & $24 / 2$ & $60 / 2$ & $-12 / 2$ & $96 / 2$ & $-48 / 2$ \\
\hline \multirow{2}{*}{60} & $76 / 6$ & $36 / 6$ & $108 / 6$ & $0 / 6$ & $144 / 6$ \\
& $48 / 4$ & $84 / 4$ & $12 / 4$ & $120 / 4$ & $-24 / 4$ \\
\hline \multirow{2}{*}{84} & $96 / 8$ & $60 / 8$ & $132 / 8$ & $24 / 8$ & $168 / 8$ \\
& $72 / 6$ & $108 / 6$ & $36 / 6$ & $144 / 6$ & $0 / 6$ \\
\hline
\end{tabular}

The number before "/" is the space order of radial electromagnetic waves, the number after "/" is a multiple of the rated frequency.

PMSM has the tendency to minimize the magnetic reluctance by adjusting the relative position between the rotor and stator during operation, which will generate the cogging torque. The fundamental frequency of the cogging torque in PMSM is the least common multiple $(\operatorname{LCM}(\mathrm{Q}, 2 \mathrm{P}))$ of the number of teeth and poles of the stator [15]. The magnetostriction will produce magnetic noise during operation of PMSM [16]. The frequency of the magnetic noise is twice the rated frequency. It can be easily solved from the main electromagnetic parameters of the 3.6 MW ECPMSDDGs that the fluctuation period of the cogging torque is 216, and the harmonic frequency of the magnetic noise is $140 \mathrm{~Hz}$ and its integer multiple.

\section{Boiling Vibration Analysis and Experiment of Two-Phase Flow in Stator Cooling Chamber}

\subsection{Analysis of Boiling Excitation Force in Two-Phase Flow}

The boiling of evaporative cooling medium in stator cooling chamber is a dynamic process of bubble formation and growth. The characteristics of two-phase flow excitation force under boiling condition are related to the inherent physical property parameters and 
the flow condition of the boiling medium. The main physical parameters of evaporative cooling medium used in 3.6 MW ECPMSDDGs are shown in Table 3.

Table 3. Main physical parameters of evaporative cooling medium.

\begin{tabular}{cc}
\hline Parameters & Value \\
\hline Boiling point $/{ }^{\circ} \mathrm{C}$ & 47.6 \\
Density $/(\mathrm{kg} / \mathrm{L})$ & 1.56 \\
Kinematic viscosity $/\left(\mathrm{mm}^{2} / \mathrm{s}\right)$ & 0.44 \\
Surface tension $/(\mathrm{mN} / \mathrm{m})$ & 17.2 \\
Coefficient of thermal expansion $/\left(1 /{ }^{\circ} \mathrm{C}\right)$ & $1.48 \times 10^{-3}$ \\
Heat conductivity coefficient $/(\mathrm{W} / \mathrm{m}-\mathrm{K})$ & 0.076 \\
Gasification latent heat $/(\mathrm{kJ} / \mathrm{kg})$ & 149.7 \\
\hline
\end{tabular}

When the uniform overheating temperature of the bubble is $\mathrm{Tf}$, the bubble grows spontaneously because the surface tension cannot balance the pressure difference between inside and outside. At this time, there is the following Equation (4):

$$
p_{g}>p_{f}+\frac{2 \sigma}{r}
$$

where $p_{g}$ is the gas pressure. $p_{f}$ is the main flow pressure. $\sigma$ represents the surface tension. $r$ is the bubble nucleation radius.

The diameter of the bubble escaping from the heating surface is determined by the equilibrium condition of the buoyancy and surface tension. The empirical formula of bubble escaping diameter modified by Cole and Shulman [17] is:

$$
D_{d}=0.0208 \beta_{c} \sqrt{\frac{\sigma}{\left(\rho_{f}-\rho_{g}\right) g}}\left[1+0.0025\left(\frac{\mathrm{d} D}{\mathrm{~d} \tau}\right)^{\frac{3}{2}}\right]
$$

where $\beta_{\mathrm{c}}$ is the contact angle. $\frac{\mathrm{d} D}{\mathrm{~d} \tau}$ is the growth rate of bubble diameter. $\rho_{g}$ is the gas density. $\rho_{f}$ is the main flow density. $g$ is the gravitational acceleration.

Considering the waiting period before bubble growth, the semi-empirical formula of bubble generation frequency modified by Zuber [18] is as follows:

$$
f_{b}=0.59\left[\frac{\sigma\left(\rho_{f}-\rho_{g}\right) g^{\frac{1}{4}}}{D_{d}}\right]^{\frac{1}{4}}
$$

where $f_{\mathrm{b}}$ is the bubble formation frequency.

Yamagata proved that the number of bubble cores $\mathrm{N}$ generated on the unit wall was closely related to the heat flux value $Q$ transferred [19], that is:

$$
n=\frac{6 q}{\pi C_{\mathrm{q}} h_{\mathrm{fg}}\left(D_{d}\right)^{3} \rho_{g} f_{\mathrm{b}}}
$$

where $n$ is the number of bubble cores generated on the heating wall unit. $q$ is the heat flux. $C_{\mathrm{q}}$ is the specific heat capacity of bubble liquid film. $h_{f g}$ is the gasification latent heat.

The limiting velocity of the bubble movement in the liquid phase is:

$$
v_{\max }=\frac{2\left(\rho_{f}-\rho_{g}\right) g D_{d}^{2}}{9 \mu_{f}}
$$

where $u_{f}$ is the flow viscosity.

Periodic oscillations would be caused by pressure waves' instability and density waves' instability of the two-phase flow under the boiling condition. The instability caused by pressure waves is high-frequency oscillation, which is related to the time that the 
pressure waves flows through the heating channel, and the oscillation frequency is usually about 10-100 Hz [20]. The instability caused by density waves is low-frequency oscillation, which is related to the time that the fluid flows through the heating channel, usually less than $1 \mathrm{~Hz}$ [21-23].

Assuming that the interface is extremely thin, the mass, momentum and energy conservation equations at the interface of two-phase flow are as follows:

$$
\begin{gathered}
\dot{m}_{k}=\rho_{k} \mathbf{n}_{k} \cdot\left(\mathbf{u}_{k}-\mathbf{u}_{i}\right) \\
\sum_{k=1}^{2} \dot{m}_{k}=0 \\
\sum_{k=1}^{2}\left[\dot{m}_{k} \mathbf{u}_{k}+\mathbf{n}_{k} \cdot \boldsymbol{\sigma}_{k}\right]+\nabla_{\beta}\left(\mathbf{t}_{\alpha} a^{\alpha \beta} \sigma\right)=0 \\
\frac{d U_{i}}{d t}+U_{\mathrm{i}} \nabla_{s} \cdot u_{i}=\sum_{k=1}^{2}\left[\dot{m}_{k}\left[U_{k}+\frac{u_{k}^{2}}{2}\right]+\right. \\
\left.\mathbf{n}_{k} \cdot\left(\boldsymbol{\sigma}_{k} \cdot \mathbf{u}_{k}+\mathbf{q}_{k}\right)\right]+\nabla_{\beta}\left(\mathbf{t}_{\alpha} a^{\alpha \beta} \sigma \cdot \mathbf{u}_{i}\right)
\end{gathered}
$$

where the subscript $k$ represents the $k$ th phase. The subscript $i$ represents the interface. $\mathbf{n}$ is the space normal vector. $\mathbf{u}$ is the velocity vector. $\boldsymbol{\sigma}$ is the stress tensor. $\mathbf{t}_{\alpha}$ is the mixed tensor between the space and the interface coordinates. $a^{\alpha \beta}$ is the interface metric tensor. $\nabla_{\beta}$ represents the interface covariant derivative. $U$ represents the per-unit energy on surface. $\nabla_{\mathrm{s}}$ represents the interface divergence. $\mathbf{q}$ is the heat flow density vector.

The flow field equations of the two-phase flow can be obtained by coupling the conservation equations of mass, momentum and energy of each phase and the interface of two-phase flow. The dynamic behavior of bubbles in two-phase flow could be solved through the initial and boundary conditions.

\subsection{Experiment on Boiling and Oscillating Characteristics of Two-Phase Flow in Stator Radial Flow Groove}

The simplified experimental model of the stator radial flow groove of 3.6 MW ECPMSDDGs is shown in Figure 3. It is difficult to identify the boiling condition of two-phase flow with the naked eye due to its complexity and instability, so the method to obtain the frequency of two-phase flow is based on two-phase oscillating image taken by high-speed camera. The two-phase flow oscillation frequency can be obtained by the bubble oscillation period obtained by shooting. It can be regarded as a period when the bubbles show the same or similar state at the same position after a period of time. The frequency of two-phase flow can be calculated according to the period.

The heat load of radial flow groove was set to $800 \mathrm{~W}$ based on the equivalent thermal effect. The bubbles oscillate mainly at the outlet and the position 1,2 and 3 are the typical boiling regions during the experimental process. It can be clearly seen that the phenomenon of bubble oscillation in experimental model is obviously different at the shooting position 1 , 2 and 3 due to the different spatial structures of the model during the experimental process. The shooting positions of positions 2 and 3 are shown in Figure 4.

Bubble oscillations photographed at three positions are shown in Figures 5-7. The oscillation periods of position 1, 2 and 3 are $0.03 \mathrm{~s}, 0.02 \mathrm{~s}$ and $0.04 \mathrm{~s}$, respectively. The oscillation frequencies of position 1, 2 and 3 are $33.3 \mathrm{~Hz}, 50 \mathrm{~Hz}$, and $25 \mathrm{~Hz}$, respectively. The experimental phenomenon showed that the oscillation frequency of position 2 is the highest during the experiment. 


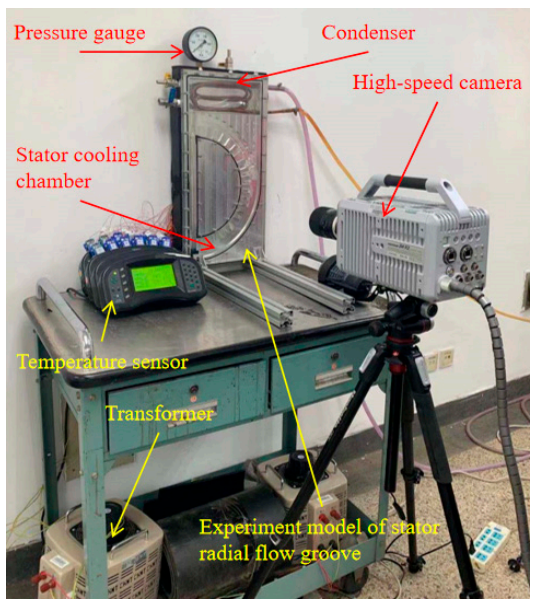

Figure 3. Experimental model of the stator radial flow groove.

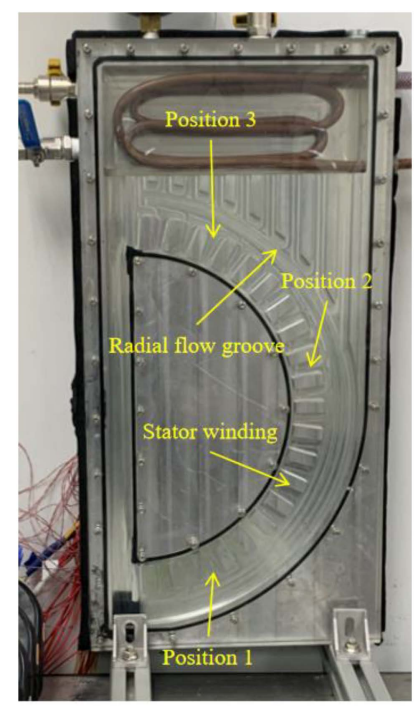

Figure 4. Experiment shooting position.

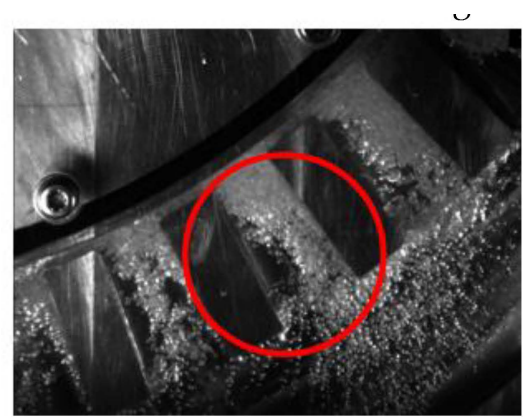

(a)

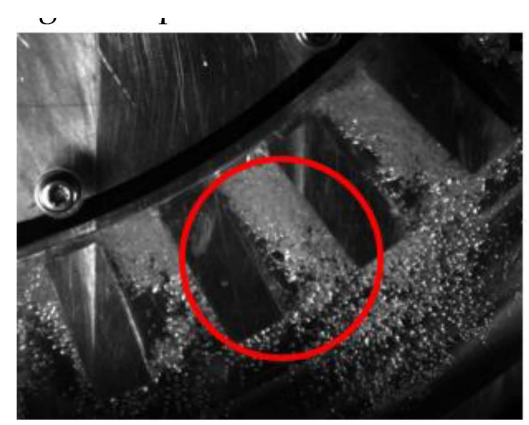

(b)

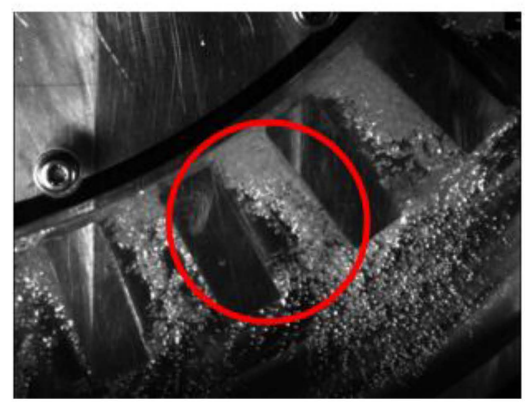

(c)

Figure 5. The two-phase flow oscillates at position 1 during one period. (a) $\mathrm{T}=0 \mathrm{~s} ;(\mathbf{b}) \mathrm{T}=0.015 \mathrm{~s} ;(\mathbf{c}) \mathrm{T}=0.03 \mathrm{~s}$.

It can be seen from the experimental results that the maximum frequency of boiling two-phase flow is about $50 \mathrm{~Hz}$ in the stator cooling chamber, which is consistent with the theory of two-phase flow boiling oscillation in which the range of two-phase flow boiling oscillation frequency is between 10 and $100 \mathrm{~Hz}$. 


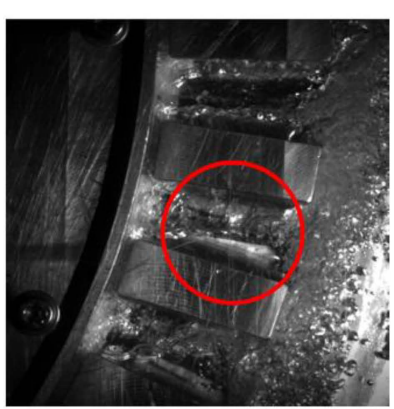

(a)

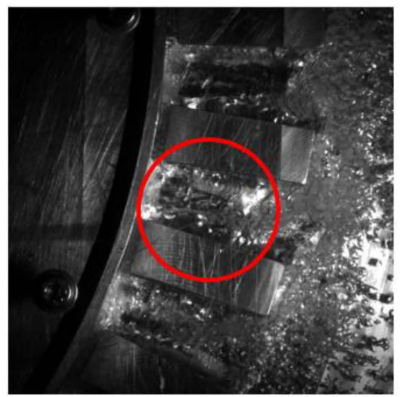

(b)

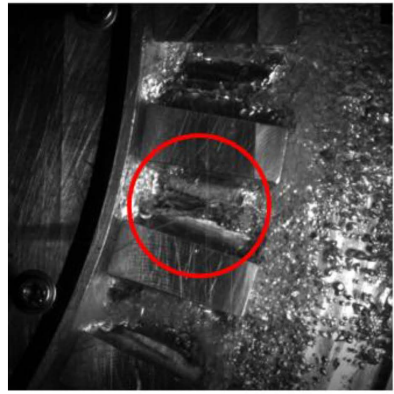

(c)

Figure 6. The two-phase flow oscillates at position 2 during one period. (a) $\mathrm{T}=0 \mathrm{~s} ;(\mathbf{b}) \mathrm{T}=0.01 \mathrm{~s}$; (c) $\mathrm{T}=0.02 \mathrm{~s}$.

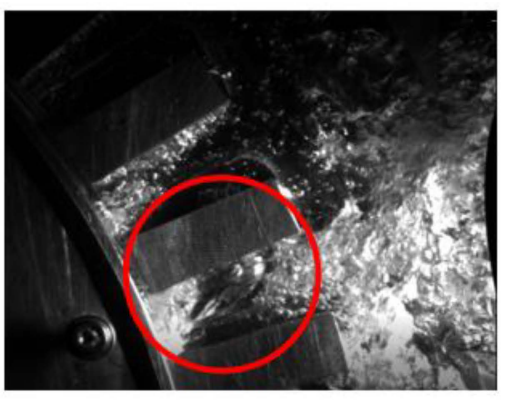

(a)

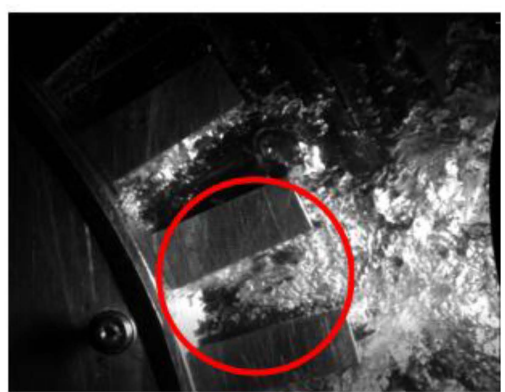

(b)

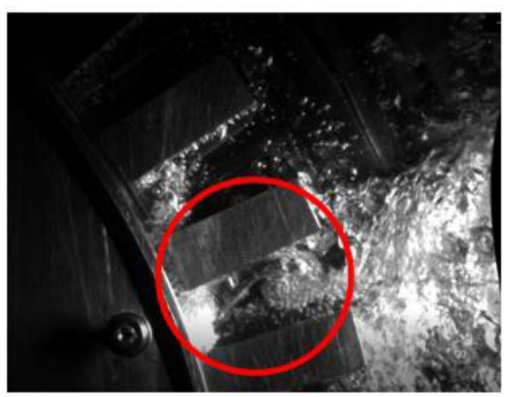

(c)

Figure 7. The two-phase flow oscillates at position 3 during one period. (a) $\mathrm{T}=0 \mathrm{~s}$; (b) $\mathrm{T}=0.02 \mathrm{~s}$; (c) $\mathrm{T}=0.04 \mathrm{~s}$.

\section{Frequency Optimization and Simulation Verification of Stator}

The motor stator will produce resonance noise when the vibration frequency of twophase flow and electromagnetic excitation force is close or equal to the natural frequency of the motor stator. Therefore, the natural vibration frequency optimization of the stator cooling structure plays an important role in the reduction of resonance noise.

\subsection{Analysis of Stator Wet Mode}

The dynamics vibration equation of stator cooling chamber is as follows:

$$
\left(\mathbf{M}_{\mathrm{s}}+\mathbf{M}_{\mathrm{f}}\right) \ddot{\mathbf{Q}}+\left(\mathbf{C}_{\mathrm{s}}+\mathbf{C}_{\mathrm{f}}\right) \ddot{\mathbf{Q}}+\left(\mathbf{K}_{\mathrm{s}}+\mathbf{K}_{\mathrm{f}}\right) \mathbf{Q}=\mathbf{G}
$$

where $\mathbf{M}_{\mathrm{s}}$ and $\mathbf{M}_{\mathrm{f}}$ represent the mass matrix of structure and fluid, respectively. $\mathbf{Q}$ represents the displacement vector. $\mathbf{C}_{\mathrm{s}}$ and $\mathbf{C}_{\mathrm{f}}$ represent the damping matrix of structure and fluid, respectively. $\mathbf{K}_{\mathrm{s}}$ and $\mathbf{K}_{\mathrm{f}}$ represent the stiffness matrix of the structure and fluid, respectively. $\mathbf{G}$ represents the force matrix.

When the motor stator is immersed in the evaporative cooling working medium, the fluid-structure coupling effect will become very obvious [24] and the natural frequency of the coupled system will be reduced [25].

The dynamics vibration equation of the fluid-structure coupling system under external excitation is as follows:

$$
\left(\left[\begin{array}{cc}
\mathbf{K}_{\mathrm{s}} & -\mathbf{S}^{\mathrm{T}} \\
0 & \mathbf{K}_{\mathrm{f}}
\end{array}\right]-\omega^{2}\left[\begin{array}{cc}
\mathbf{M}_{\mathbf{S}} & 0 \\
\mathbf{S} & \mathbf{M}_{\mathrm{f}}
\end{array}\right]\right)\left[\begin{array}{l}
\mathbf{d} \\
\mathbf{p}
\end{array}\right]=\left[\begin{array}{c}
\mathbf{f}_{\mathrm{S}} \\
\mathbf{f}_{\mathrm{f}}
\end{array}\right]
$$

where $\mathbf{S}$ represents the fluid-structure coupling matrix. $\omega$ represents the vibration frequency. $\mathbf{d}$ represents the structure vibration displacement matrix. $\mathbf{p}$ represents the flow field sound pressure matrix. $\mathbf{f}_{\mathrm{f}}$ and $\mathbf{f}_{\mathrm{s}}$ represent the excitation force matrix of fluid and structure, respectively. 
When the fluid-structure coupling field is without any outside excitation, the sound pressure distribution of the flow field is as follows:

$$
\left(\mathbf{K}_{\mathrm{f}}-\omega^{2} \mathbf{M}_{\mathrm{f}}\right) \mathbf{p}=\omega^{2} \mathbf{S d}
$$

The structural coupling matrix will become:

$$
\left[\mathbf{K}_{\mathrm{s}}-\omega^{2} \mathbf{M}_{\mathrm{s}}-\omega^{2} \mathbf{S}^{\mathrm{T}}\left(\mathbf{K}_{\mathrm{f}}-\omega^{2} \mathbf{M}_{\mathrm{f}}\right)^{-1} \mathbf{S}\right] \mathbf{d}=\mathbf{f}_{\mathrm{s}}
$$

The wet mode analysis of the motor stator of 3.6 MW ECPMSDDGs was carried out by workbench ACT in ANSYS software. The two-phase flow was considered as acoustic fluid in the analysis and the sound velocity in the fluid field was set to be $1300 \mathrm{~m} / \mathrm{s}$. The radial flow groove of the stator was supported by I-beam, so fixed constraints were applied to the axial supporting beam. The stator was pressed into the motor frame and fitted with the front end cover, so fixed constraints were applied to the stator end. Material properties for simulation were defined as follows: the Poisson's ratio, density and elastic modulus of the silicon steel sheet was defined as $0.26,7850 \mathrm{~kg} / \mathrm{m}^{3}$ and $200 \mathrm{GPa}$, respectively. The Poisson's ratio, density and elastic modulus of the structure steel was defined as $0.25,7850 \mathrm{~kg} / \mathrm{m}^{3}$ and $206 \mathrm{GPa}$, respectively. The finite element meshing size was set to be $10 \mathrm{~mm}$ and the total number of finite elements was 1,834,244. The self-weight load was applied in the wet modal analysis of the structure. The wet modes analysis results of the stator core are shown in Figure 8.

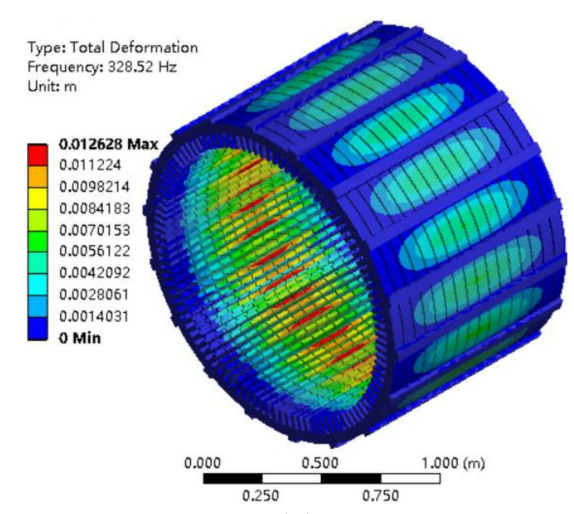

(a)

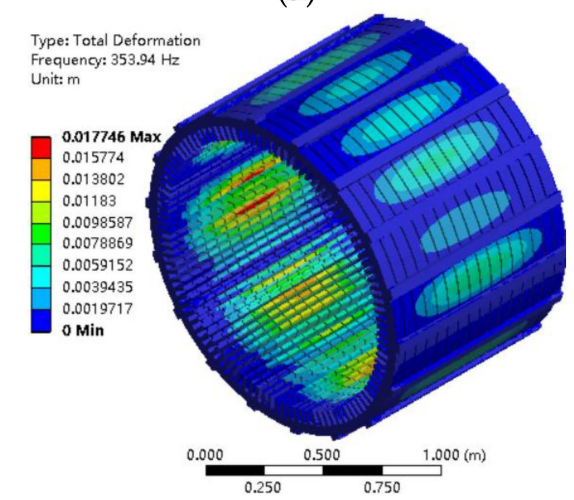

(d)

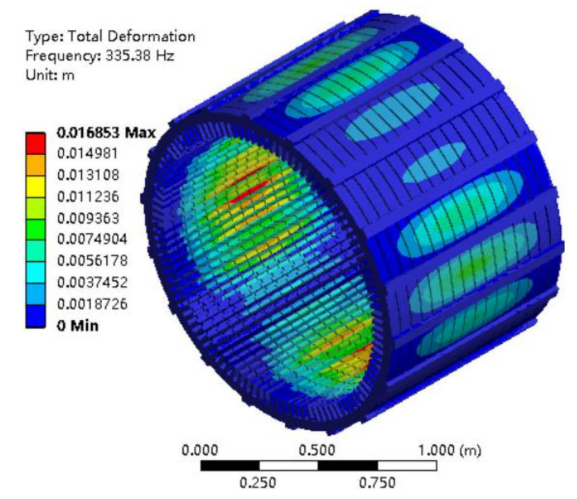

(b)

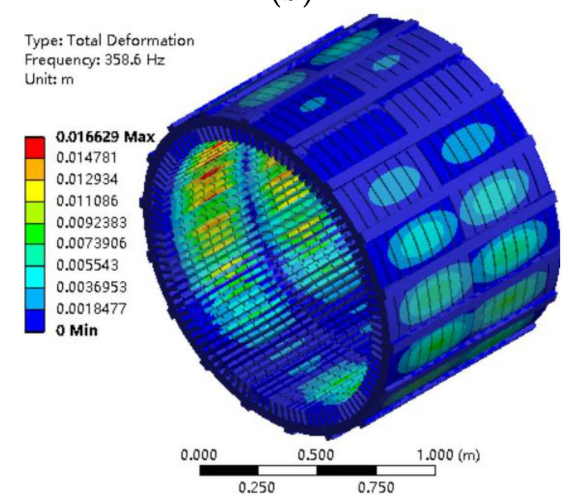

(e)

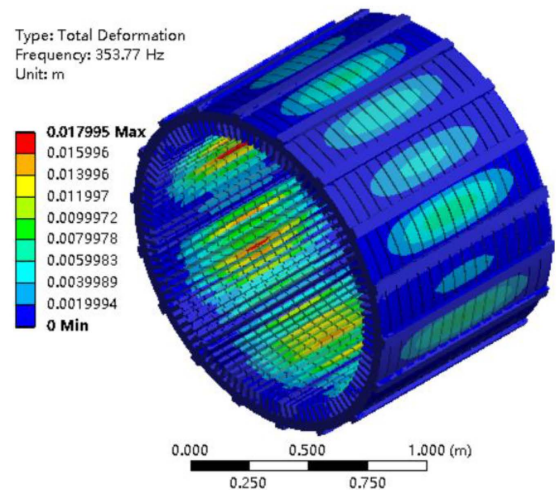

(c)

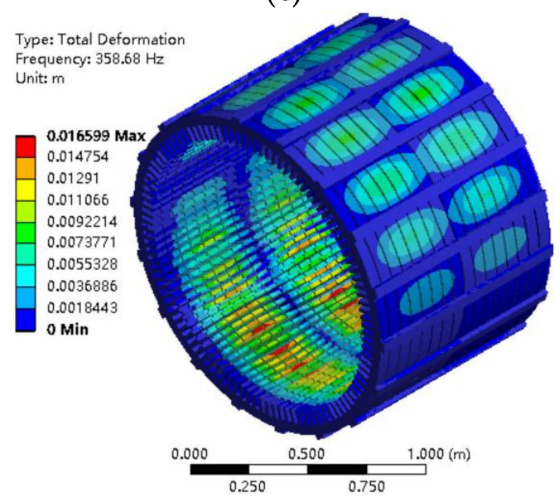

(f)

Figure 8. The first six frequencies and modes of the stator core wet mode. (a) The first mode shape diagram; (b) the second mode shape diagram; (c) the third mode shape diagram; (d) the fourth mode shape diagram; (e) the fifth mode shape diagram; (f) the sixth mode shape diagram.

The continuum has an infinite number of mode shapes, which are arranged in ascending order to be first, second and third modes based on modal frequencies. A very small excitation input can cause a large amplitude of vibration (resonance) at the natural 
frequency, but the transmission of excitation through the system is reduced with the increase of the natural frequency, so the higher order responses of the structure could be ignored. It can be seen from the wet mode analysis results that the natural frequencies of the low order wet mode of the stator are not too high and some natural frequencies are close to the vibration frequencies of the electromagnetic exciting force. The low order wet mode natural frequencies of the stator core should be increased to avoid the possibility of resonance caused by both two-phase flow and electromagnetic excitation force in the frequency optimization.

\subsection{Frequency Optimization of the Stator Structure}

The frequency optimization was based on topology optimization. The natural frequency of stator structure could be changed by modifying the density of structural units in the structure. In order to identify the best position of structural units modification for each step during the optimization process, the sensitivity analysis is needed for each unit during the optimization. The unit frequency sensitivity could be obtained based on FEA. The undamped vibration equation of the structure is:

$$
\left([\mathbf{K}]-\omega_{f}^{2}[\mathbf{M}]\right) \mathbf{u}_{f}=0
$$

where $\mathbf{K}$ is the total stiffness matrix. $\mathbf{M}$ is the total mass matrix. $\omega_{f}$ is the inherent frequency of fth order. $\mathbf{u}_{f}$ is the feature vector that corresponds to $\omega_{f}$.

When the density in the ith unit of the structure is modified, the change amount of the structural frequency caused by this modification can be approximately calculated as follows:

$$
\Delta\left(\omega_{f}^{2}\right)=\frac{1}{m_{f}}\left\{\mathbf{u}_{j}^{i}\right\}^{T}\left(\omega_{f}^{2}\left[\mathbf{M}^{i}\right]-\left[\mathbf{K}^{i}\right]\right)\left\{\mathbf{u}_{j}^{i}\right\}
$$

where $m_{f}$ is the modal mass. $\mathbf{u}_{j}^{i}$ is the vector only associated with element $i$ in $\mathbf{u}_{f}$. $\left[\mathbf{M}^{i}\right]$ is the mass matrix for element $i$. $\left[\mathbf{K}^{i}\right]$ is the stiffness matrix for element $i$.

The natural vibration frequency of the optimized structure can be adjusted to the expected value by reducing the density of less sensitive units during the optimization process.

The structure optimization of the motor stator of 3.6 MW ECPMSDDGs was carried out by topology optimization in ANSYS software. Structural topology optimization is used to optimize the topology shape of the structure to meet the requirements of the conditions. The optimization objective was to maximize the first order natural vibration frequency of the wet mode of the stator core. The constraint condition is volume constraint. Frequency optimization of stator structure was divided into two parts: structure optimization of the stator core supporting beam and the radial flow groove. The optimized region and non-optimized region of the stator core supporting beam are shown in Figure 9.

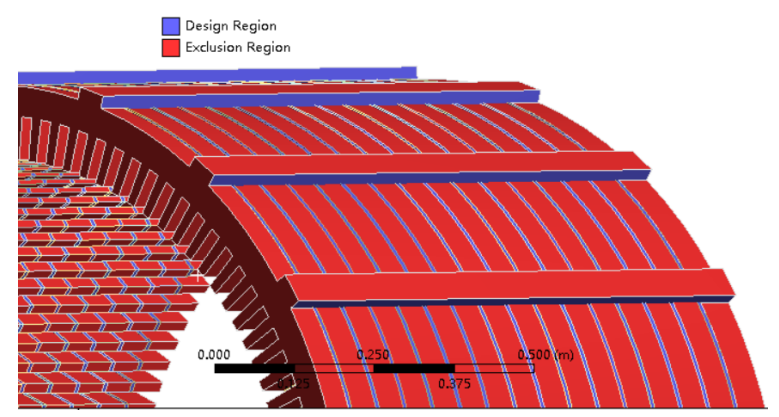

Figure 9. Optimization region and non-optimization region of stator core supporting beam.

Fixed constraints were imposed on the upper surface of the radial beam and the end face of the stator core. The volume optimization constraint was set at $70 \%$ and the optimization results of the radial beam are shown in Figure 10 after 30 iterations: 


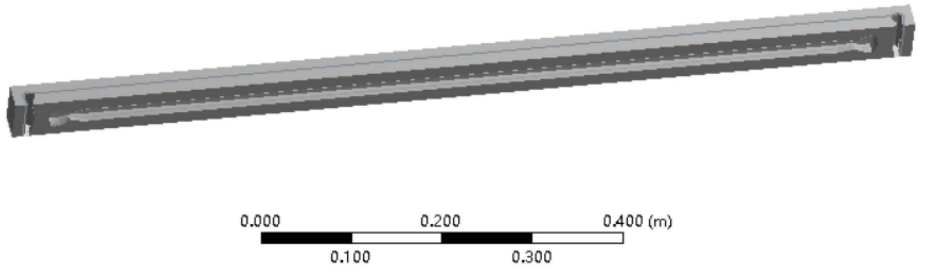

Figure 10. Frequency optimization result of stator core supporting beam.

It can be considered that the heat dissipation capacity and electromagnetic performance of the motor can remain the same under the condition that the length of the radial flow groove of the permanent magnet motor remains constant. The optimized region and non-optimized region of the stator core radial flow groove are shown in Figure 11.

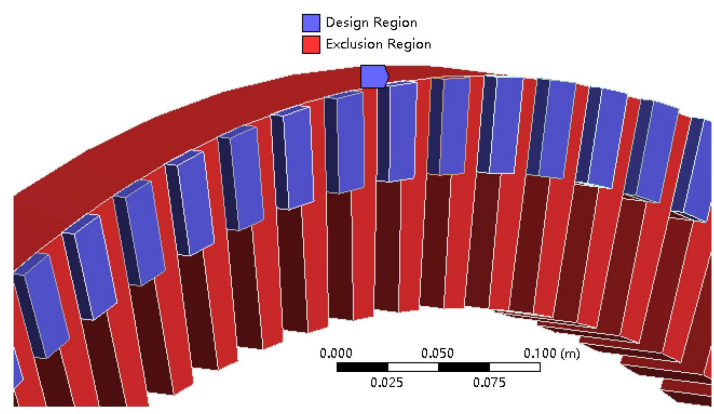

Figure 11. Optimization area and non-optimization area of the stator core radial flow groove.

The volume optimization constraint was set at $70 \%$ and the optimization results of the radial flow groove are shown in Figure 12 after 30 iterations.

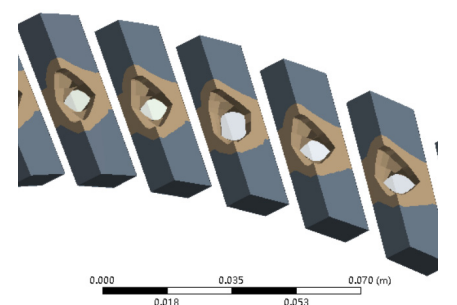

Figure 12. Frequency optimization result of stator radial flow groove.

Due to the process and manufacturing requirements, the optimized structure of the stator radial flow groove was changed to the form shown in Figure 13.

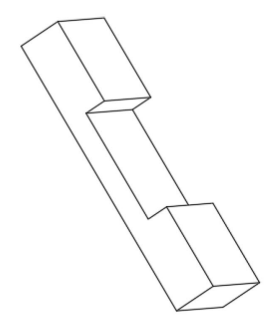

Figure 13. Optimization result of radial flow groove.

The wet mode natural frequency of the optimized stator structure was simulated based on FEA. The natural frequency of the stator can be improved by changing the structure shape of the radial flow groove and the supporting beam. The first six order frequencies and modes of the wet mode of the optimized stator structure are shown in Figure 14. It can be seen from the comparison between Figures 8 and 14 that the first six wet mode 
natural frequencies of the stator structure after optimization have been improved. The lowest frequency of the first wet mode has been increased from $328.52 \mathrm{~Hz}$ to $985.99 \mathrm{~Hz}$ after optimization, which could effectively avoid the low order resonance vibration caused by electromagnetic and two-phase flow excitation force.

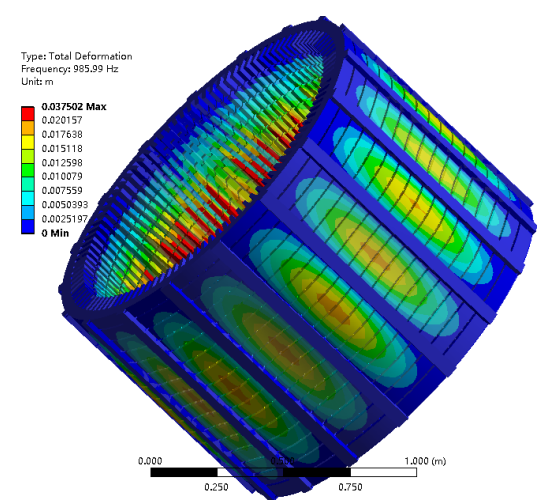

(a)

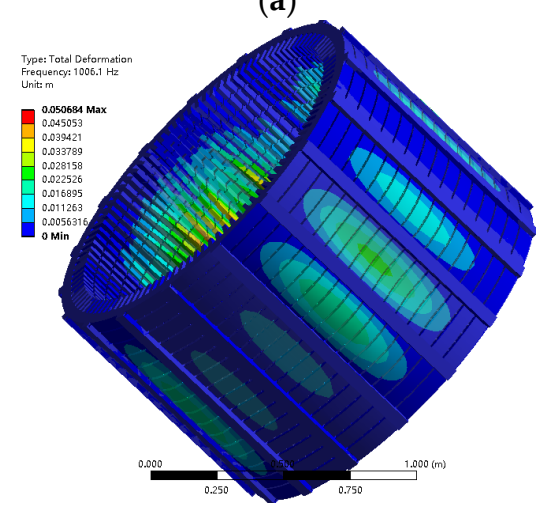

(d)

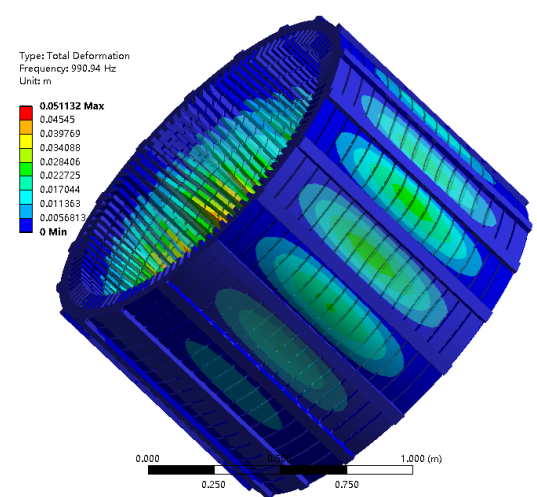

(b)

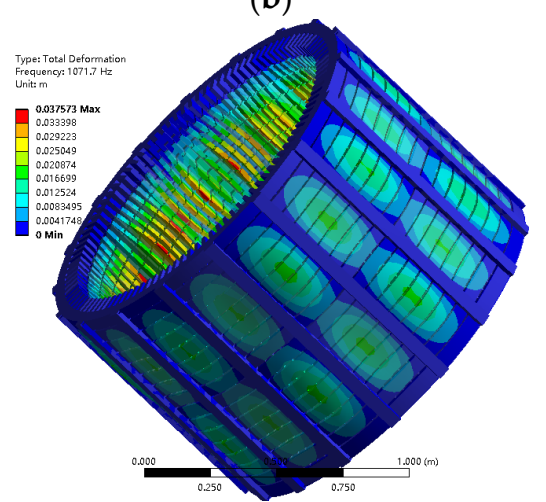

(e)

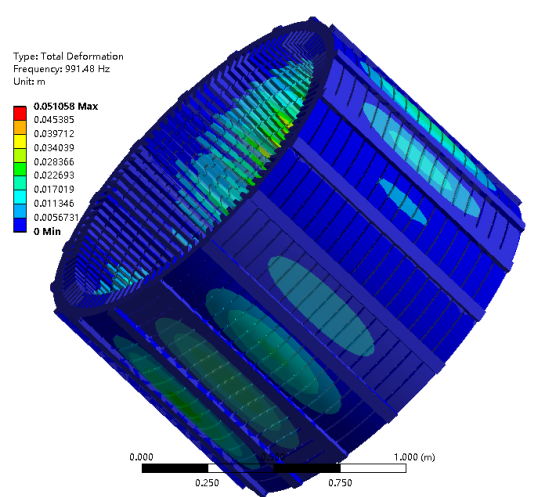

(c)

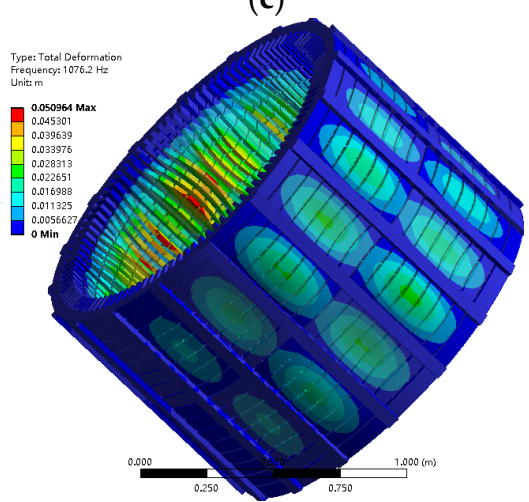

(f)

Figure 14. The first six frequencies and modes of the optimized stator core wet mode. (a) The first mode shape diagram; (b) the second mode shape diagram; (c) the third mode shape diagram; (d) the fourth mode shape diagram; (e) the fifth mode shape diagram; (f) the sixth mode shape diagram.

\subsection{Motor Noise Simulation}

The noise analysis of the motor stator of 3.6 MW ECPMSDDGs was carried out by workbench ACT in ANSYS software based on multiphysical field coupling. It was assumed that the two-phase flow boiling and electromagnetic excitation force independently leads to the vibration and noise of the motor during the operation. The noise of the motor caused by electromagnetic and two-phase flow separately is obtained by coupling two-phase fluid field simulated by Fluent software and the electromagnetic field simulated by Maxwell software with workbench ACT in ANSYS, respectively. For two-phase flow simulation, the working medium and the motor are modeled separately and the interfaces are set as the fluid-solid coupling interface. Copper loss is set at $102 \mathrm{~kW}$ and iron loss is set at $18 \mathrm{~kW}$ during two-phase flow boiling simulation. The properties of the cooling medium are set in accordance with those properties in Table 3 . The mesh is divided by hexahedral elements and the total number of finite elements is $4,587,430$. The simulation is based on evaporative and condensation model and the saturation temperature is set to be $320 \mathrm{~K}$. After iteration and stabilization, the 1/4 simplified model of two-phase flow simulation in stator cavity is shown in Figure 15 and the excitation force of two-phase flow can be transferred to workbench multi-physical field coupling by Fluent software. Finally, the noise analysis was carried out by coupling the excitation force of two-phase flow from Fluent software and the structural modal analysis results with the pre-established air areas in workbench ACT module. 


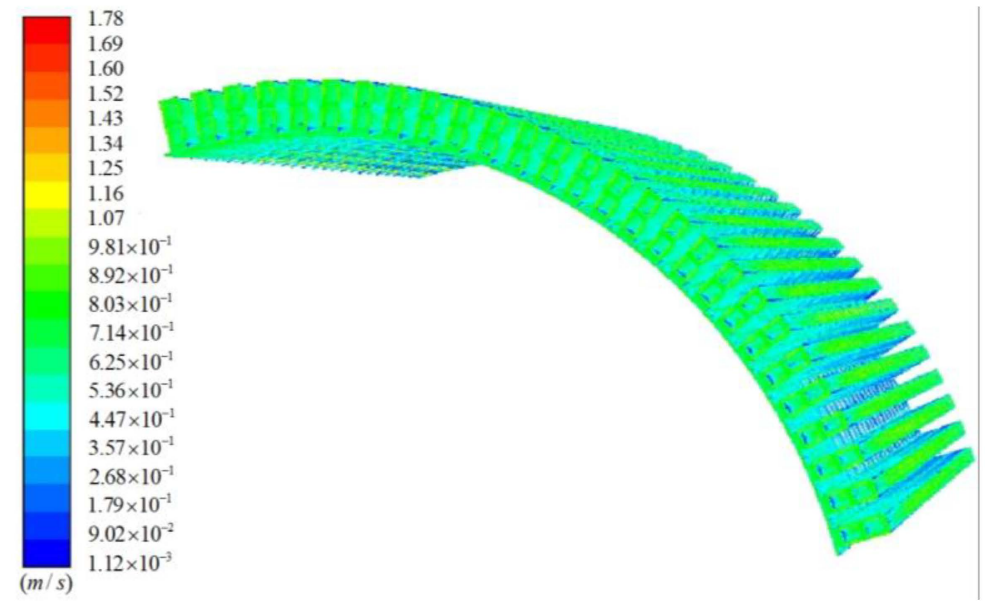

Figure 15. Two-phase flow simulation of velocity distribution in stator cavity.

For electromagnetic simulation, the current source excitation is adopted in the simulation of electromagnetic field of the motor under rated load. The classical mesh division is adopted in electromagnetic field simulation. The electromagnetic force simulation is based on the two-dimensional transient magnetic solver. After iteration and stabilization, the noise analysis was carried out by coupling the electromagnetic excitation force from Maxwell software and the structural modal analysis results with the pre-established air areas in workbench ACT module.

The frequency response spectrum of the equivalent radiated power of stator outside surface solely caused by electromagnetic and two-phase flow exciting force was analyzed based on the coupling simulation of harmonic response module in workbench software, respectively. The solution range of the frequency is set to be $0-1500 \mathrm{~Hz}$ and the solution method is set to be full solution method. The boundary conditions of the response are directly imported by Maxwell and Fluent module, respectively. The frequency interval is set to be $30 \mathrm{~Hz}$. The comparison diagrams of frequency response spectrum before and after optimization are shown in Figures 16 and 17.

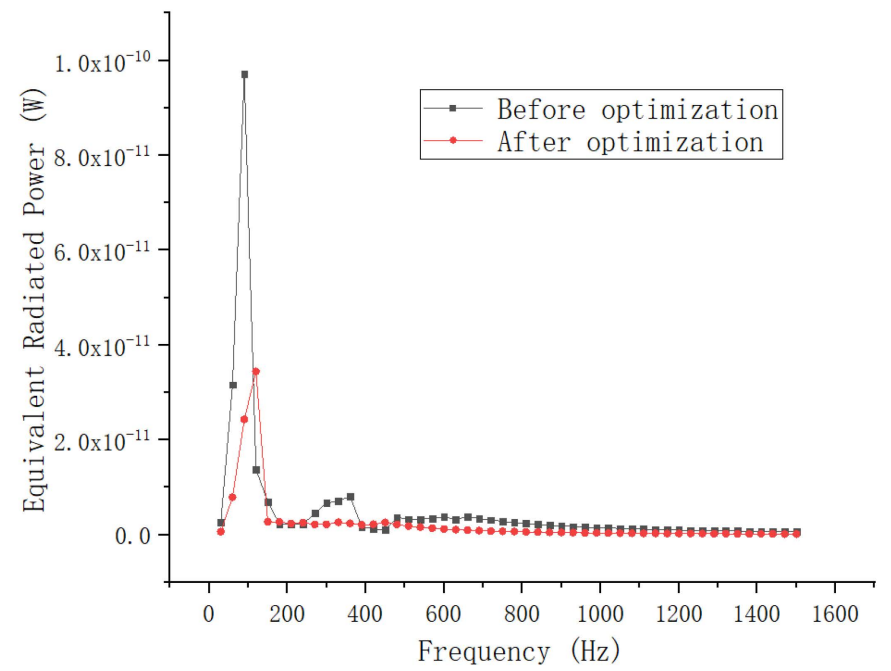

Figure 16. Comparison of equivalent radiated power induced by two-phase flow excitation force. 


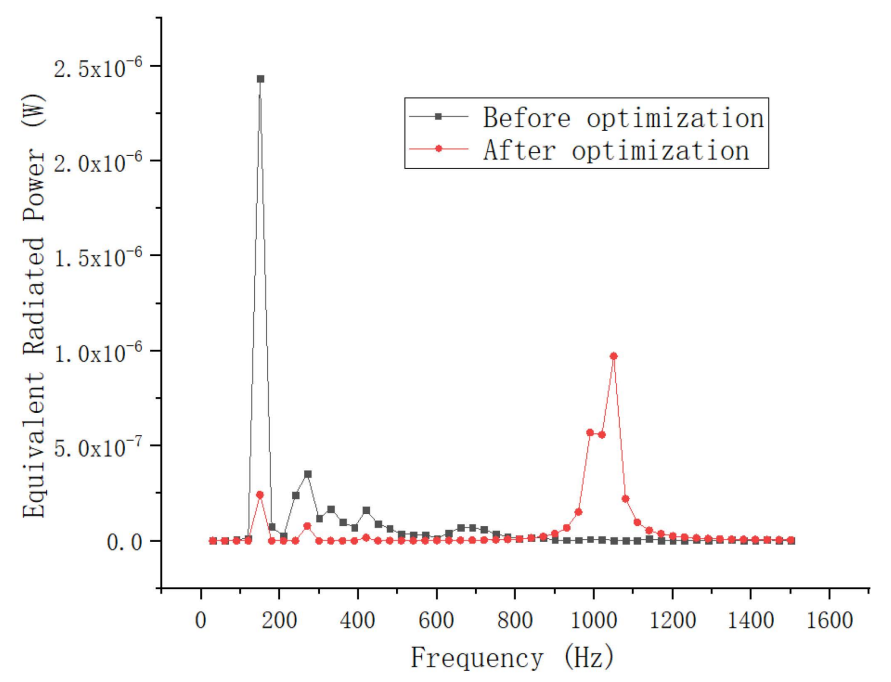

Figure 17. Comparison of equivalent radiated power induced by electromagnetic excitation force.

It can be seen from the comparison that the equivalent radiated power of the stator outside surface has been reduced in the low frequency region after optimization and the resonance frequency of optimized structure has been improved. The two-phase flow excitation force mainly produced low frequency vibration and noise which could be reduced by improving the first natural frequency of the structure. The vibration and noise caused by the electromagnetic force in the low frequency region could be reduced by the optimized structure and transferred to the resonance vibration with smaller amplitude in the high frequency region.

The resonance noise of the motor stator solely caused by the two-phase flow boiling and electromagnetic excitation force is shown in Figures 18 and 19. The sound in liquid has much higher sound pressure levels then in gas in Figure 19 due to its higher sound speed.

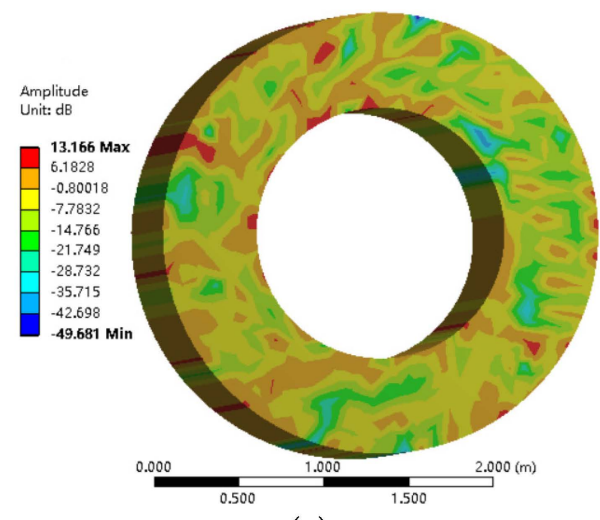

(a)

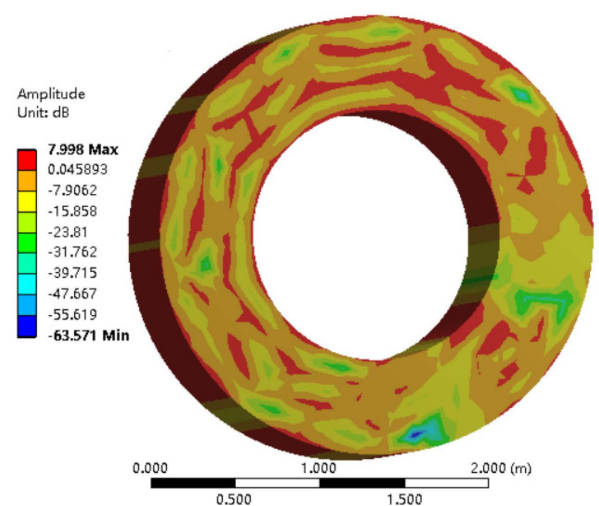

(b)

Figure 18. Comparison diagram of stator core noise caused by two-phase flow excitation force before and after optimization.

(a) Noise distribution of the surrounding area of the original stator core caused by the excitation force of two-phase flow;

(b) noise distribution of the surrounding area of the optimized stator core caused by the excitation force of two-phase flow.

It can be seen from the analysis and comparison that the noise of the motor stator solely caused by electromagnetic and two-phase flow resonance could be reduced after the stator cooling structure optimization. The results showed that the optimized stator cooling structure could reduce the noise of the motor under the rated operating conditions. 


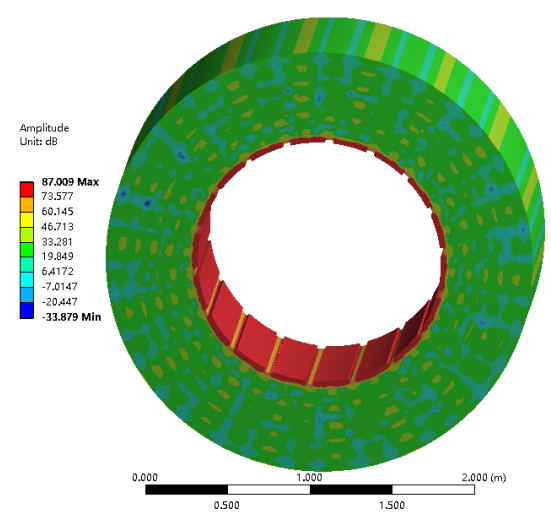

(a)

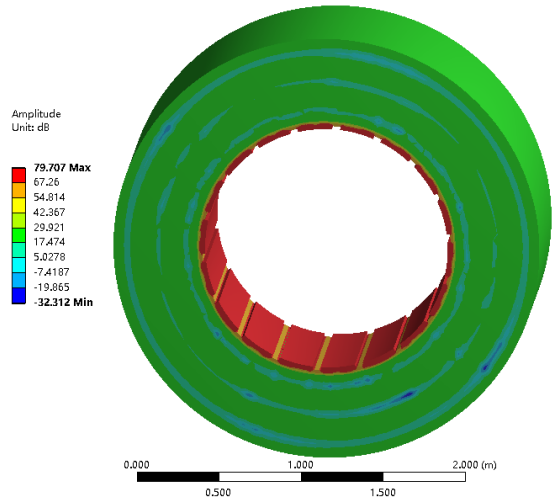

(b)

Figure 19. Comparison diagram of stator core noise caused by electromagnetic excitation force before and after optimization. (a) Noise distribution of the surrounding area of the original stator core caused by the electromagnetic excitation force; $(\mathbf{b})$ noise distribution of the surrounding area of the optimized stator core caused by the electromagnetic excitation force.

\section{Discussion}

Different from the traditional motor, the vibration of ECPMSDDGs stator cavity would be affected by the boiling of two-phase flow and electromagnetic force. The oscillations of the two-phase flow at each position of stator cavity are different from each other due to the characteristics of two-phase flow varying with the occurrence of different conditions. The electromagnetic force contributes more to the vibration of the stator because its higher frequency is closer to the motor wet natural vibration frequency, while the boiling force of two-phase flow contributes less to the low-order vibration because of its lower frequency compared with the electromagnetic force. The resonance caused by the two-phase flow and the electromagnetic force should be considered at the same time during the process of noise optimization.

Different from the motor noise optimization design in other articles, the wet modal natural frequency of the stator could be improved without changing the structure of the stator through optimizing the radial flow groove and the supporting beam of the evaporative cooling motor. The resonance noise induced by low frequency excitation force could be reduced by increasing the first order natural frequency of the structure.

\section{Conclusions}

The stator optimization process was proposed in this paper to reduce resonance vibration and noise caused by electromagnetic and two-phase flow. The two-phase flow and electromagnetic force vibration sources were considered in this research. The main vibration frequencies of electromagnetic and two-phase flow in stator cooling chamber were analyzed and the vibration frequency distribution of two-phase boiling force was obtained by the shooting experiment. The first six wet mode natural vibration frequencies of the stator were obtained based on fluid-structure coupling analysis. The wet mode natural frequency of the stator cooling structure of 3.6 MW ECPMSDDGs was improved based on the stator cooling structure optimization. The FEA simulation verified that the natural frequency of stator was improved after optimization and the noise simulation based on FEA proved that optimized structure could reduce the resonance noise caused by electromagnetic and two-phase flow. This optimization process could not only provide a new idea for the design and optimization of the evaporative cooling motor but also could provide a reference for the motor design and optimization process under other working conditions. The prospect of reducing motor noise by changing the type and amount of evaporative cooling medium or the effect of cooling structure on the vibration of two-phase flow could be studied in the future. 
Author Contributions: Writing-review and editing, Z.C.; project administration, L.R.; funding acquisition, S.H.; data curation, J.Y. All authors have read and agreed to the published version of the manuscript.

Funding: This research was funded by National Natural Science Foundation of China (51737004).

Institutional Review Board Statement: Not applicable.

Informed Consent Statement: Not applicable.

Data Availability Statement: The study did not report any data.

Acknowledgments: High-speed camera used for experiments; associate researcher Chen Jinxiu for bubble filming assistance; associate researcher Xiong Bin for experimental platform designing; associate professor Gao Jian for theoretical supporting; postgraduate student Feng Yun for experimental platform and cooling devices building assistance; postgraduate student Zhao Yufeng for photo processing assistance.

Conflicts of Interest: The authors declare no conflict of interest. The funders had no role in the design of the study; in the collection, analyses, or interpretation of data; in the writing of the manuscript, or in the decision to publish the results.

\section{References}

1. Yaramasu, V.; Wu, B.; Sen, P.C.; Kouro, S.; Narimani, M. High-power wind energy conversion systems: State-of-the-art and emerging technologies. Proc. IEEE 2015, 103, 740-788. [CrossRef]

2. Guobiao, G.; Lin, R. Applications and Developments of the Evaporative Cooling Technology in the Field of Hydrogenerators. Proc. CSEE 2014, 34, 5112-5119.

3. Deng, W.; Zuo, S. Electromagnetic vibration and noise of the permanent-magnet synchronous motors for electric vehicles: An overview. IEEE Trans. Transp. Electrif. 2018, 5, 59-70. [CrossRef]

4. Seryakov, A.V. Resonant vibration heat transfer coefficient increase of short low-temperature heat pipes. Int. J. Heat Mass Transf. 2020, 158, 119764. [CrossRef]

5. Unno, N.; Yuki, K.; Taniguchi, J.; Satake, S.I. Boiling heat transfer enhancement by self-excited vibration. Int. J. Heat Mass Transf. 2020, 153, 119588. [CrossRef]

6. Elkholy, A.; Kempers, R. Experimental investigation of geyser boiling in a small diameter two-phase loop thermosyphon. Exp. Therm. Fluid Sci. 2020, 118, 110170. [CrossRef]

7. Lin, F.; Zuo, S.G.; Deng, W.Z.; Wu, S.L. Reduction of vibration and acoustic noise in permanent magnet synchronous motor by optimizing magnetic forces. J. Sound Vib. 2018, 429, 193-205. [CrossRef]

8. Islam, M.S.; Islam, R.; Sebastian, T. Noise and Vibration Characteristics of Permanent-Magnet Synchronous Motors Using Electromagnetic and Structural Analyses. IEEE Trans. Ind. Appl. 2014, 50, 3214-3222. [CrossRef]

9. Latoufis, K.; Matzakos, A.; Katsambiris, I.; Vassilakis, A.; Hatziargyriou, N. Acoustic noise of axial flux permanent magnet generators in locally manufactured small wind turbines. IET Renew. Power Gener. 2019, 13, 2922-2928. [CrossRef]

10. Islam, J.; Svechkarenko, D.; Chin, R.; Szucs, A.; Mantere, J.; Sakki, R. Cogging torque and vibration analysis of a direct-driven PM wind generator with concentrated and distributed windings. In Proceedings of the 15th International Conference on Electrical Machines and Systems (ICEMS), Sapporo, Japan, 21-24 October 2012; IEEE: Piscataway, NJ, USA, 2012; pp. 1-6.

11. Shinagam, R.; Ajay, G.; Patta, L.; Gandam, A.S. Vibration and noise studies on wind turbine generator for reduction of vibrations and noise. World J. Eng. 2020, 39, 693-702. [CrossRef]

12. Zhu, H.; Yoshida, S.; Kajiwara, H.; Ogawa, Y.; Nakada, S.; Ono, J. A study on torsional vibration reduction for variable-speed variable-pitch wind turbines. In Proceedings of the 2017 IEEE International Conference on Mechatronics (ICM), Gippsland, Australia, 14-17 February 2017; IEEE: Piscataway, NJ, USA, 2017; pp. 318-323.

13. Taegen, F.; Kolbe, J.; Verma, S.P. Vibrations and noise produced by special purpose permanent-magnet synchronous motors in variable frequency operation. In Proceedings of the 4th IEEE International Conference on Power Electronics and Drive Systems. IEEE PEDS 2001-Indonesia. Proceedings (Cat. No. 01TH8594), Denpasar, Indonesia, 25 October 2001; IEEE: Piscataway, NJ, USA, 2001; Volume 2, pp. 583-588.

14. Islam, R.; Husain, I. Analytical model for predicting noise and vibration in permanent-magnet synchronous motors. IEEE Trans. Ind. Appl. 2010, 46, 2346-2354. [CrossRef]

15. Deng, A.Q.L.; Hong, B.S.; Xiao, C.F. Influence of design parameters on cogging torque in directly driven permanent magnet synchronous wind generators. In Proceedings of the 2009 International Conference on Electrical Machines and Systems, Miami, FL, USA, 3-6 May 2009; IEEE: Piscataway, NJ, USA, 2009; pp. 1-5.

16. Somkun, S.; Moses, A.J.; Anderson, P.I. Effect of magnetostriction anisotropy in nonoriented electrical steels on deformation of induction motor stator cores. IEEE Trans. Magn. 2009, 45, 4744-4747. [CrossRef]

17. Cole, R.; Shulman, H.L. Bubble departure diameters at subatmospheric pressures. Chem. Eng. Prog. Symp. Ser. 1966, 62, 6-16. 
18. Zuber, N. Hydrodynamic Aspects of Boiling Heat Transfer; No. 4439; United States Atomic Energy Commission, Technical Information Service: Alexandria, VA, USA, 1959.

19. Yamagata, K.; Hirano, F.; Nishikawa, K.; Matsuoka, H. Nucleate boiling of water on the horizontal heating surface. Mem. Fac. Eng. Kyushu 1955, 15, 98.

20. Bergles, A.E.; Lopina, R.F.; Fiori, M.P. Critical-heat-flux and flow-pattern observations for low-pressure water flowing in tubes. J. Heat Transf. 1967, 89, 69-74. [CrossRef]

21. Sun, Q.; Yang, R.; Zhao, H. Predictive study of the incipient point of net vapor generation in low-flow subcooled boiling. Nucl. Eng. Des. 2003, 225, 249-256. [CrossRef]

22. Jeng, H.R.; Pan, C. Analysis of two-phase flow characteristics in a natural circulation loop using the drift-flux model taking flow pattern change and subcooled boiling into consideration. Ann. Nucl. Energy 1999, 26, 1227-1251. [CrossRef]

23. Pettigrew, M.J.; Taylor, C.E.; Kim, B.S. Vibration of tube bundles in two-phase cross-flow: Part 1-Hydrodynamic mass and damping. J. Press. Vessel. Technol 1989, 111, 466-477. [CrossRef]

24. Rodriguez, C.G.; Egusquiza, E.; Escaler, X.; Liang, Q.W.; Avellan, F. Experimental investigation of added mass effects on a Francis turbine runner in still water. J. Fluids Struct. 2006, 22, 699-712. [CrossRef]

25. Egusquiza, E.; Valero, C.; Liang, Q.; Coussirat, M.; Seidel, U. Fluid added mass effect in the modal response of a pump-turbine impeller. In Proceedings of the International Design Engineering Technical Conferences and Computers and Information in Engineering Conference, San Diego, CA, USA, 30 August-2 September 2009; Volume 48982, pp. 715-724. 